\title{
Calor: Una Propuesta Didáctica Constructivista con Enfoque de Ciencia Integrada
}

(Heat: a constructivist didactic proposal with integrated science focus)

\author{
Alberto de L. Castillo, Dora I. Granados, Luis A. Marino \\ Departamento de Ciencias Naturales, Facultad de Humanidades y Ciencias, \\ Universidad Nacional del Litoral, Ciudad Universitaria, Paraje el Pozo S/N. \\ C.P. 3000, Santa Fe, Argentina, \\ E-Mails: adlcastillo@ciudad.com.ar-favantgranados@infovia.com.ar
}

Recebido em 27 de novembro, 2001. Aceito em 19 de março, 2002.

\begin{abstract}
Realizamos un estudio comparativo entre dos diseños didácticos constructivistas para la enseñanza del tema Calor en estudiantes de carreras en ciencias naturales. Uno con un enfoque de ciencia integrada y el otro con un enfoque no integrador. En el primer diseño el alumno construye su propio conocimiento resolviendo situaciones problemáticas relacionadas a la biología y el medio-ambiente, que le permite analizar y relacionar los conceptos involucrados, mientras que en el segundo construye su conocimiento a través de la resolución de situaciones problemáticas físicas pero no biológicas. Concluimos que el primer diseño favorece en los alumnos la construcción de una estructura cognitiva del tipo integrada, que les facilita internalizar y transferir los conceptos físicos a problemas concretos de su especialidad.
\end{abstract}

We made a comparative study between two constructivists didactic design, for teaching the theme Heat in students of natural sciences careers. One with a integrated science focus and another with a non integrator focus. In the first design the student could build his own understanding, by solving problematical situations related to the biology and the natural environment, that allows him to analyze how are related the involucrated concepts, while in the second he build his understanding by solving physics but non biological problematic situations. We concluded that the first design allows to build in the students an cognitive structure of integrated type, wath makes it easier for them to internalize and transfer the physical concepts to problems of his specialty.

\section{Introducción}

Una problemática observada en las aulas desde la experiencia docente, refiere a la asiduidad de encontrar dificultades en integrar los conceptos relativos a la física, con los de otras areas de aplicación. Por ejemplo, en el caso de los alumnos de carreras en ciencias naturales con importantes lineamientos ecologistas en materia curricular, la dificultad se manifiesta a la hora de integrar los conceptos físicos elementales (impartidos en una física básica) con aquellos relacionados a los fenómenos biológicos, el medioambiente y sus problemáticas.

Esta fragmentación del conocimiento le trae al alumno problemas al momento de aplicar los conocimientos físicos a asignaturas posteriores, e incluso al momento de enfrentarse con situaciones problemáticas concretas de tipo profesional. Es decir en definitiva que se genera un déficit de transferencia.

\section{Fundamentación de la pro- puesta}

Nuestra propuesta se basa en el desarrollo de los distintos temas de una Física General presente en la currícula de carreras en ciencias naturales, con cierta carga en temas ecologístas, en base a un marco teórico que por un lado se sustenta en los lineamientos psicológicos y metodológicos de la enseñanza constructivista[1],[2], [3], [4], [5], y por el otro en el enfoque de ciencia integrada [6], [7], es decir, integrar los conceptos y las formas de pensar de las ciencias involucradas en un fenómeno desde la introducción del tema, hasta la resolución y análisis de situaciones problemáticas asociadas a él. Al respecto tambien coincidimos con que urge encontrar fórmulas 
de integración y coordinación posibles y eficientes de cara a posibilitar a los alumnos una aproximación a los temas científicos, los avances en las areas de sus competencias y las implicancias sociales que ellos conllevan[8].

Con respecto a este último punto, los defensores de la ciencia integrada argumentan que este enfoque explicita más claramente lo que es común a todas las ciencias y permite una comprensión más global de los fenómenos naturales[6].

Además, partimos de la idea de que cualquiera sea la estrategia de enseñanza-aprendizaje utilizada en el dictado de una clase de ciencias, el curriculum de esta funciona como una bisagra entre el discurso natural de sentido común y el discurso no natural de la investigación científica[9]. Por esta razón es necesario plantear situaciones de la vida cotidiana, que para su comprensión requieran transitar hacia la conceptualización científica.

Proponemos activar y articular los conocimientos previos de los alumnos con distintas problemáticas relacionadas a fenómenos biológicos, a través del incentivo que significa el poder relacionarlos a la física, guiándolos hacia la construcción de una concepción científica del tema en estudio, utilizando dichos conocimientos para efectuar análisis reflexivos sobre otros campos de las ciencias naturales, las distintas problemáticas de los ambientes naturales y sus posibles implicancias; facilitando la integración e internalización de dichos conceptos, con lo cual esperamos que su aprendizaje resulte significativo.

\section{Implementación didáctica}

Con el fín de estudiar la eficacia de nuestra propuesta se efectuó un estudio no experimental comparativo entre 2 grupos de 30 alumnos cursantes de la asignatura Física General de las carreras Profesorado en Biología y Licenciatura en Biodiversidad. En uno de los mismos implementamos nuestra propuesta de enseñanza constructivista con el enfoque integrador del tipo biológicoambiental, mientras que en el otro tomado como "testigo" se utilizó la estrategia de enseñanza tradicional, no integradora, usada en el curso de Física General, ambas aplicados al tema de "Calor, temperatura, y los fenómenos asociados".

Este tema se eligió porque tiene conceptos físicos (calor y temperatura) que presentan una relación especial con la biología, y permiten plantear interesantes relaciones con ella. Además poseen su origen en nociones intuitivas basadas en la interpretación de estímulos sensoriales. Por lo tanto su estudio formal debe partir de un esfuerzo de interpretación y modelización de di- chos estímulos y sensaciones, por lo cual es sumamente útil para estudiar la evolución en la estructura conceptual del alumno.

\section{A. Conformación de los grupos}

La población completa de alumnos era de 85 alumnos, cuyas edades oscilaban entre los 18 y 19 años. De dicha población se seleccionó una muestra de 60 alumnos en base a las siguientes pautas:

1- Nivel de abstracción para la resolución de problemas. (Se seleccionaron alumnos que habían cursado y/o rendido Química General e Inorgánica, en la cual se requiere modelizar mentalmente en una forma similar a Física ). 2- Manejo de herramientas matemáticas. 3Disponibilidad de tiempo.

A su vez los 2 grupos a estudiar se armaron y homogeneizaron teniendo en cuenta :

1- Formación previa: Se refiere a la procedencia de los alumnos. Se homogeneizaron los grupos ubicando en cada grupo el mismo porcentaje de alumnos procedentes de cada terminalidad secundaria ( bachiller, técnica, o bachiller con orientación biológica). 2- Base conceptual previa (en cuanto a conceptos) similar. Para esto se entregaron una serie de fenomenologías, en base a los conocimientos sensoriales que habitualmente tienen los alumnos sobre el tema [10]. De las mismas se plantearon interrogantes con respuestas de opción múltiple, con una opción correcta y 3 distractores.

Se evaluaron las respuestas correctas (asignándoles 1 pt a cada una) y se empleó la fórmula de Horts para efectuar una corrección por una posible adivinación en las respuestas[11]:

$$
P c=C-E /(n-1)
$$

En donde Pc: Puntaje corregido; C: Puntaje original; $\mathrm{E}$ : Nro de respuestas erróneas; $\mathrm{n}$ : Nro total de alternativas de cada Ítem.

Los 2 grupos se conformaron de modo que el puntaje total de cada grupo fuese el mismo.

Además al final de cada fenomenología se pidió a cada alumno la elaboración de un mapa conceptual que permitió analizar las pequeñas unidades sistémicas que conformaban su estructura concetual previa sobre el tema.

Por ejemplo para analizar los conceptos de calor, temperatura, malos y buenos conductores del calor se presentó la siguiente fenomenología:

"Al tomar un trozo de metal con la mano lo siento "frío", pero si tomo un trozo de madera (ambos a la intemperie) no". Al respecto señale la opción que crea correcta: a) El hierro es un buen conductor del calor..... 
b) El hierro es un mal conductor del calor..... c) La madera es buena conductora del calor..... d) La madera es mala conductora del calor .....

Si recubro el hierro y la madera por una tela, observo: a) Que el fenómeno ya no se nota..... b) Que el fenómeno sigue invariable..... c) Que el fenómeno se invierte..... d) Ninguna de las anteriores.....

En definitiva a que atribuiría Ud.. el fenómeno original: a) A que hay un mayor flujo de calor de la mano al hierro que de la mano a la madera.... b) A que no hay flujo de calor de la mano a la madera ..... c) A que hay flujo de frío desde el hierro a la mano ..... d) Ninguna de las anteriores.....

En cuanto a las temperaturas podría concluír que: a) La temperatura del hierro es menor a la de la madera..... b) No existe diferencia de temperatura entre la madera y la mano..... c) La mano está a mayor temperatura que el hierro o la madera .....

d) Ninguna de las anteriores.....

Elabore en base a sus respuestas anteriores un mapa conceptual, ubicando los conceptos con que explica el fenómeno según un orden jerárquico por ud. seleccionado, explicitando las relaciones existentes entre ellos.

\section{B. Temas a desarrollar}

Calor y temperatura.- Dilatación de los cuerpos.Mecanismos de transferencia de calor.- Modelo de Fourier para la conducción; Convección natural y forzada; Radiación; Modelo de cuerpo negro.- Procesos de cambios de fase basados en modelos cinéticos-moleculares.

\section{Estrategias didácticas implementadas}

Referencias: A.D. $=$ Actividad Docente./ A.A.= Actividad del alumnado.

\section{Estrategia constructivista con enfoque inte- grador}

Etapa a). A.D.: * Introducción temática. * Organización y discusión de los conceptos teóricos básicos en base a la estructura conceptual previa de los alumnos. * Proposición de situaciones biológicas y/o ambientales con base física, que ponen en conflicto la estructura conceptual previa errónea de los alumnos. * Proposición y coordinación de actividades reflexivas y de integración. A.A.:* Reflexión de situaciones problemáticas físicas con un enfoque integrador del tipo biológico y/o ambiental, relativas al tema "Calor...".

Etapa b). A.D.: * Facilitar el acceso a las fuentes de información bibliográficas (ver[12], [13], [14], [15], [16], [17], [18], [19], audiovisual, informático (cedido por Greenpeace), e infografías sobre temas ecológicos.
* Moderación de los debates grupales. A.A.: * Resolución en forma grupal de situaciones problemáticas cualitativas integradas del tipo biológica y/o ambiental (Ver Anexo A).

* Investigación del material informativo. * Intercambio de ideas entre los integrantes del grupo y elaboración de síntesis explicativas.

Etapa c). A.D.: * Moderación y orientación de los debates intergrupales.A.A.: * Elaboración grupal de argumentos explicativos. * Presentación grupal de las argumentaciones. * Debates e intercambios de ideas en forma intergrupal.

Etapa d). A.D.: * Moderación en el proceso de generación de conclusiones generales y corroboración de las leyes físicas. A.A.: * Elaboración intergrupal de conclusiones generales y clara presentación de las leyes físicas que las sustentan. * Información de los resultados.

Etapa e) ó de transferencia final. A.A.: * Mecanismos de termorregulación de los homotermos. (Física Biológica, Zoología). * Efecto Invernadero. (Ecología). * Hábitos migracionales de especies marinas (Zoología).

\section{Estrategia constructivista sin enfoque inte- grador}

Etapa a). A.D.: * Introducción temática. * Organización y discusión de los conceptos teóricos básicos en base a la estructura conceptual previa de los alumnos. * Proposición de situaciones físicas naturales (biológicas o no), que ponen en conflicto los preconceptos erróneos de los alumnos. * Proposición y coordinación de actividades reflexivas. A.A.: * Reflexión de situaciones problemáticas físicas con ejemplos académicos y/o naturales, relativas al tema "Calor...".

Etapa b). A.D.: * Facilitar el acceso a las fuentes de información bibliográficas (ver[20], [15], [16], [18], [21]), audiovisual, e informático (acceso a páginas web sobre el tema de calor). * Moderación de los debates grupales. A.A.: Resolución en forma grupal de situaciones problemáticas cualitativas del tipo académicas, y naturales (biológicas ó no) (Ver Anexo B). * Investigación del material informativo. ${ }^{*}$ Intercambio de ideas entre los integrantes del grupo y elaboración de síntesis explicativas.

Etapa c). A.D.: * Moderación y orientación de los debates intergrupales.

A.A.: * Elaboración grupal de argumentos explicativos. * Presentación grupal de las argumentaciones. * Debates e intercambios de ideas en forma intergrupal.

Etapa d). A.D.: * Moderación en el proceso de generación de conclusiones generales y corroboración de 
las leyes físicas. A.A.: * Elaboración intergrupal de conclusiones generales y clara presentación de las leyes físicas que las sustentan.

Etapa e). A.A.: * Comunicación de los resultados y reflexiones epistemológicas.

\section{Instrumento de medición utilizado}

Para evaluar el impacto que la estrategia constructivista con enfoque biológico-ambiental tuvo sobre el aprendizaje significativo de los alumnos y su estructura cognitiva, se utilizaron cuestiones fenomenológicas extraídas de diarios ó revistas científicas.

Dichas cuestiones permitieron trabajar con problemas cualitativos y abiertos, los cuales proporcionaron un ámbito muy útil para analizar la capacidad reflexiva del alumno y sus modos de pensar (metaconocimiento). Se entregó un problema por alumno y se fijó un plazo de 1 día para que verbalize por escrito las respuestas, siguiendo una serie de premisas destinadas a permitirnos analizar las variables y categorías sometidas a análisis.

Actividad. Para el siguiente problema, explicitar claramente los siguientes puntos:

1- Identificación del problema (planteo del fenómeno en término de variables físicas) y el modelo físico asociado a él. 2- Explicitación de las hipótesis resolutivas que fijen el marco de contorno del modelo a utilizar para la resolución del problema. 3- Elaboración de una respuesta justificada en base a un texto argumentativo, con el auxilio de representaciones gráficas y/ó el empleo de expresiones matemáticas que la sustente. 4- a) Explicitación de los conceptos físicos, biológicos y ambientales asociados al fenómeno. b) Clasificación de los conceptos en un orden de inclusión, dandole el número 1 al más abarcativo, y una numeración decreciente para los menos inclusivos. c) Explicitar las relaciones que pueda hallar entre los conceptos, clasificándolas en relaciones físicas-físicas, físicas-biológicas, físicas-ambientales y biológicas ambientales. d) Elaboración de un mapa conceptual en donde se visualize la información anterior.

Ejemplos de problemas: Problema 1 "Según las estadísticas compiladas desde el año 1905, los veranos de Santa Fe en los cuales se han registrado las mayores temperaturas veraniegas, se corresponden con aquellos años en los cuales el río Paraná a la altura del dique 2 del puerto ha registrado los menores niveles ( menos de $3 \mathrm{~m}$ ), además según los datos registrados a partir de 1970, en dichas fechas la diferencia entre las sensaciones térmicas y las temperaturas máximas resultan ser mínimas" (Fuente: Diario El Litoral de Santa Fe). Cual es el papel del río en la termorregulación del ambiente? Por qué se reducen las diferencias entre la tempe- ratura real y la sensación térmica al estar bajo el nivel del río?.

Problema 2 "Los camarones de mar poco profundo ascienden en grandes nubes que pueden llegar a varios metros cúbicos, por encima de rocas ubicadas en el fondo del mar. Estas rocas siempre gozan de la luz solar, sin embargo a pesar de esto en su migración ascencional la nube de camarones trata de escapar de la luz directa del sol para alcanzar las proximidades de la superficie y comenzar su descenso migracional hacia las rocas asoleadas del fondo" ( Fuente: American Association for the advancement of Science). Por qué la nube de camarones se forma solamente sobre las rocas soleadas? Si es por la temperatura, entonces por qué evitan la luz directa del sol? Finalmente, por qué descienden nuevamente al fondo completando una especie de "rueda de la fortuna" ?.

\section{Variables y categorías anali- zadas}

1- Identificación del modelo teórico. Significa interpretar el fenómeno desde el punto de vista de una teoría física determinada. Al respecto podemos citar las siguientes categorías: a) Uso de un marco teórico no reconocido para el planteo del problema: los alumnos recurrieron a sus preconceptos (muchos de ellos protocientíficos) para plantear el problema propuesto. b) Modelo teórico erróneo: Correspondieron a aquellos casos en los que los alumnos asociaron el problema con otro semejante ya resuelto o con el que estaban familiarizado (criterio de semejanza), por ejemplo emplear el modelo de evaporación de sustancias puras para el caso de soluciones.

c) Modelo teórico correcto: corresponde a los casos de encuadre del problema dentro del marco teórico correcto desde un punto de vista físico.

2- Formulación de las hipótesis resolutivas. Están intimamente relacionadas con establecer los límites de validez del modelo, de acuerdo al problema planteado. En este caso es el alumno quien debe fijarlas. Las categorías encontradas son: a) No hay formulación concreta: esto significa que los alumnos no fijaron los límites del modelo ni pudieron aplicarlo correctamente en las condiciones paras las que fué propuesto; Por ej. el aire es un buen aislante térmico desde el punto de vista de la conducción pero no de la convección. b) Utilización intuitiva pero no explícita: a los alumnos que resolvieron de este modo el problema, esto les restó capacidad para realizar predicciones u otro tipo de extrapolaciones. c) Formulación correcta y concreta: corresponde a los casos en que los alumnos demostraron 
poder fijar y conocer los límites de validez del modelo, lo cual les permitió realizar extrapolaciones, relaciones con otros fenómenos u otro tipo de inferencias.

3- Justificaciones: según señala Waftorsky [22] "Explicar algo es haber llegado a entenderlo de tal manera que uno sea capaz de hacer que otro lo entienda". Veslin[23] recoge la definición de explicación que da Lliboutry para quien: "Explicar consiste, en general, en reducir un hecho acontecido a un cierto nivel de complejidad, a un nivel de organización y complejidad inmediatamente inferior; Por ej. pasar de las propiedades de la célula a la fisicoquímica de las membranas celulares". Consideramos a la justificación como la estrategia utilizada para llevar a cabo dicha explicación y la argumentación, el modo de comunicarla.(ya sea verbalmente, a través de un texto, o por medio de una serie de ecuaciones, como ocurre en los problemas matemáticos).

$\mathrm{Al}$ respecto encontramos las siguientes categorías: a) Desconexión entre el fenómeno y el marco teórico asociado a él: En estos casos los alumnos no pudieron establecer una secuencia lógica que conectara el fenómeno con los conceptos asociados al modelo, ya sea porque no identificaron acabadamente el problema, porque no entendieron los conceptos asociados al modelo, ó porque simplemente no pudieron vincular ambos (modelización del problema) por poseer deficiencias para realizar la abstracción necesaria. b) Justificaciones de tipo personales. En estos casos los alumnos recurrieron a argumentaciones de tipo personales usualmente de tipo tautológicas o basadas en sus concepciones o experiencias previas.

c) Justificaciones de acuerdo al modelo y sus hipótesis implícitas. Algunos alumnos poseen el nivel cognitivo que les permitió modelar adecuadamente el problema en términos de variables físicas y establecer la secuencia necesaria para asociar el fenómeno con la explicación en términos de las mismas.

4- Estructura cognitiva de los alumnos: "Si entendemos la estructura cognitiva de un individuo, en una cierta área de conocimiento, como el contenido y la organización conceptual de sus ideas en esa área, los mapas conceptuales pueden ser utilizados para representar la estructura cognitiva del alumno"[24]. Primero se analizaron los conceptos pertinentes al fenómeno analizado (a), discriminándoselos en físicos $\left(\mathrm{a}_{1}\right)$, biológicos $\left(\mathrm{a}_{2}\right)$ y ambientales $\left(\mathrm{a}_{3}\right)$. Posteriormente se analizaron las conexiones correctamente establecidas (b), discriminadas en físicas-físicas $\left(b_{1}\right)$, físicas-biológicas $\left(b_{2}\right)$, físicas-ambientales $\left(\mathrm{b}_{3}\right)$ y biológicas-ambientales $\left(\mathrm{b}_{4}\right)$ y luego en base al análisis del mapa conceptual se consideraron las jerarquizaciones correctas establecidas entre las conceptos anteriores (c).

\section{Resultados obtenidos}

Los resultados en forma de porcentajes grupales, se representan graficamente (Fig. 1, Fig. 2, Fig. 3 y Fig. $4)$.

Debido a lo reducido de la población y a las condiciones de contorno prefijadas para formar los grupos, consideramos adecuado no efectuar ninguna extrapolación estadística que pudiera llevarnos a resultados no representativos.

$V_{1}$ ) Para las variables 1,2 y 3 y sus categorías, los porcentajes grupales se obtuvieron en base al cociente entre el número de respuestas que se encuadran en cada categoría y el número total de respuestas de cada grupo.

$\mathrm{V}_{2}$ ) Para la variable 4 y sus categorías a, b y c primero se evaluaron los $\%$ positivos individuales en base al cociente entre el número correcto, respectivamente, de conceptos, conexiones y jerarquías, y el número total de conceptos conexiones y jerarquías planteados por cada alumno. Luego en cada grupo se promediaron los $\%$ de todos sus integrantes para obtener un $\%$ representativo grupal.

Para las subcategorías $\mathrm{a}_{1}, \mathrm{a}_{2}$ y $\mathrm{a}_{3}$ primero se evaluaron los cocientes porcentuales en base al cociente entre el número correcto, respectivamente, de conceptos físicos, biológicos y ambientales, y el máximo poblacional correcto por categoría en el grupo. Finalmente se evaluó el \% representativo grupal a través del promedio de los \% de todos los integrantes.

Para las subcategorías $b_{1}, b_{2}, b_{3}, y b_{4}$ se procedió del mismo modo que en el punto anterior pero teniendo en cuenta las relaciones entre los conceptos físicos- físicos, físicos- biológicos, físicos ambientales y biológicos-ambientales.

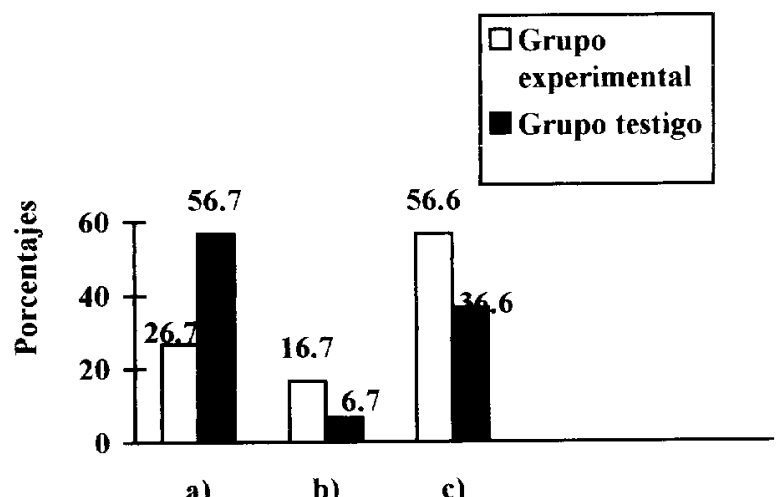

Figura 1. Identificacón del modélo teórico. En donde: a) Uso de un marco teórico no reconocido; b) Modelo teórico erróneo; c) Modelo teórico correcto. 


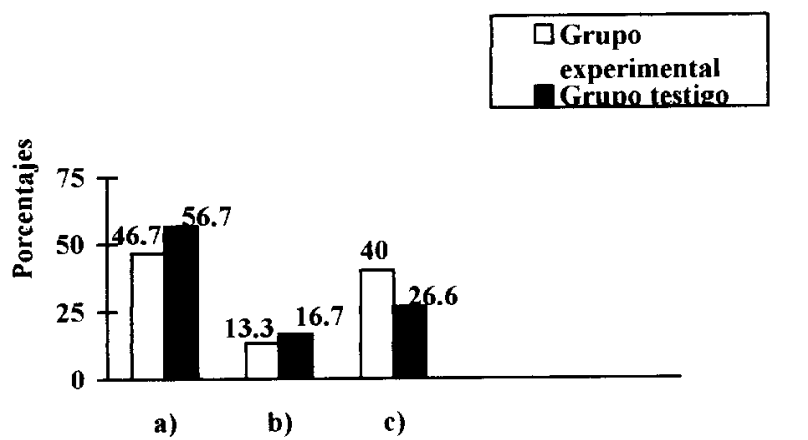

Figura 2. Formulación de las hipótesis resolutivas. En donde: a) Sin formulación concreta; b) Utilización intuitiva pero no explícita; c) Formulación correcta y concreta.

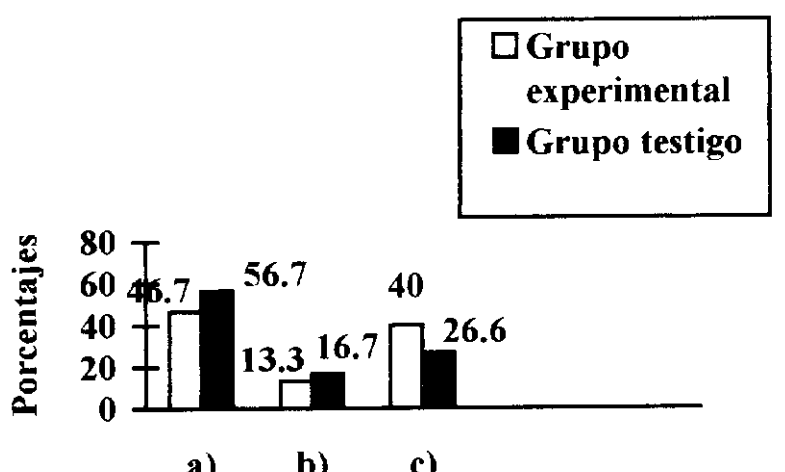

Figura 3. Gráfica comparativa de las Justificaciones utilizadas. En donde a)Desconexión entre el fenómeno y el marco teórico asociado a él; b) Justificaciones personales; c) Justificaciones de acuerdo al modelo y sus hipótesis implícitas.

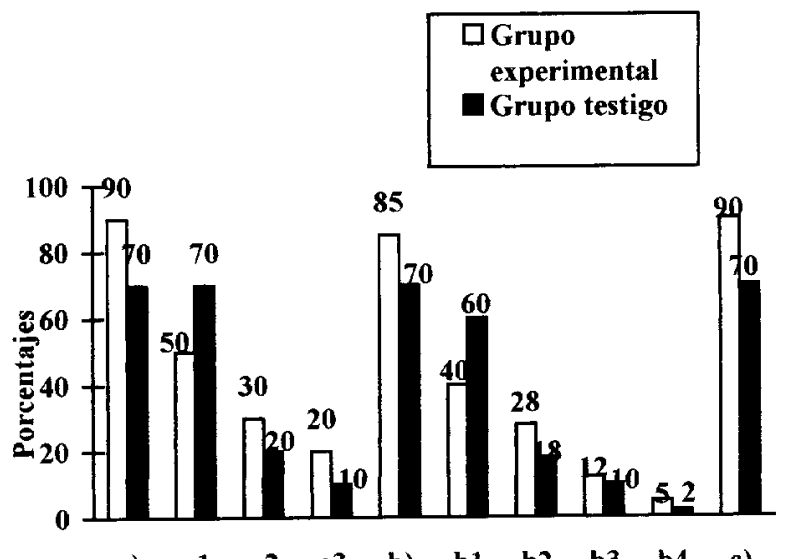

$\begin{array}{llllllllll}\text { a) } & \text { a1 } & \text { a2 } & \text { a3 } & \text { b) } & \text { b1 } & \text { b2 } & \text { b3 } & \text { b4 } & \text { c) }\end{array}$

Figura 4. Gráfica comparativa del análisis de la estructura cognitiva. En donde: a) Conceptos pertinentes al fenómeno analizado, discriminado en: $a_{1}$ ) Físicos, $a_{2}$ ) Biológicos y $a_{3}$ ) Ambientales. b) Conexiones conceptuales correctamente establecidas, discriminadas en: $b_{1}$ ) FísicasFísicas, $\left.b_{2}\right)$ Físicas-Biológicas, $b_{3}$ ) Físicas-Ambientales y $\left.b_{4}\right)$ Biológicas-Ambientales. C) Jerarquizaciones correctas.

\section{Conclusiones}

De los resultados obtenidos podemos inferir las siguientes tendencias, a ser confirmadas por futuras experiencias:

* La propuesta integradora permitió a los alumnos conectar no sólo conceptos físicos, con los de otras disciplinas, sino conocer también la forma de pensar y trabajar en las mismas.

* A consecuencia de lo anterior se mejoró la transferencia de los conceptos físicos a otras áreas como la biología y la ecología (a través de el estudio de fenómenos ambientales), con lo cual el alumno internalizó mejor dichos conceptos y el aprendizaje resultó más significativo.

* El introducir los conceptos básicos a través del planteo de problemáticas naturales del ambiente en el cual el alumno se ve inmerso y que ponen en conflicto sus concepciones erróneas, favorece el cambio conceptual.

* Através del análisis de los mapas conceptuales se observa que mediante esta propuesta integradora los alumnos adquieren una estructura cognitiva "más integrada" del tema, lo cual se pone de manifiesto en las numerosas relaciones "transversales" planteadas entre los conceptos de las distintas áreas, los cuales no son analizados como campos aislados de conocimiento.

* Podemos terminar afirmando que elaborar un curriculum, sobre la base de actividades (en este caso áulicas) integradas es factible; Esto ofrece a los docentes oportunidad para perfeccionar su práctica específica a través de la interacción con especialistas de otras disciplinas para elaborar distintos aspectos de una misma situación abordadas desde una óptica globalizadora y no acotada por una única disciplina.

\section{Referências}

[1] Ausubel, Novac y Hanesian, Psicología Educativa, Ed. Trillas, México, (1986).

[2] Novac J. D., Aprendiendo a aprender, Ed. Martinez Roca, Barcelona, (1988).

[3] Pozo J. I., Teorías cognitivas del aprendizaje, Ed. Morata, Madrid, (1989).

[4] Litwin E, Debates Constructivistas, Ed. Aique, Buenos Aires, (1998).

[5] Camilloni A. R. y otros, Corrientes didácticas contemporáneas, Ed. Paidós, Buenos Aires, (1999).

[6] Gil Perez y otros, Propuesta de secuenciación en ciencias de la naturaleza, Ed. Escuela Española, Madrid, (1993).

[7] Aranega y otros, Coordinación e integración de disciplinas, FaMAF- UNC, Córdoba (Argentina), (1994).

[8] Saéz Beames M.J. y Carretero A.J., Rev. de Ed., 310, 43-62, (1996).

[9] Wolper L., La naturaleza no natural de la ciencia, Ed. Acento, Madrid, (1994). 
[10] Iparraguirre L.M. y otros, Rev. de Enseñanza. de la Física, 11, 39-51, (1998).

[11] Beltrán F., Algunas ideas sobre la metodología de la enseñanza de la Química, Ed. Magisterio, Buenos Aires, (1984).

[12] Cicardo V.H., Biofísica, Lopez libreros editores, Buenos Aires, $6^{\text {ta }}$ Ed. (1975).

[13] Cromer A.H., Física para las ciencias de la vida, Ed. Reverté S.A., Barcelona, (1984).

[14] Glynn H.J. y Heinke G.W., Ingeniería Ambiental, Ed. Prentice Hall, México, (1996).

[15] Hewitt P.G., Conceptos de Física, Ed. Limusa, México, (1999).

[16] Jou D. y otros, Física para ciencias de la vida, Ed. La Colina, España, (1986).

[17] Shonle J.I., Environmental applications of general physics, Ed. Addison-Wesley, Delawere, (1974).

[18] Strother G.K., Física aplicada a las ciencias de la salud, Ed. Mc Graw-Hill, Bogotá, (1980).

[19] Walker J., La Feria Ambulante de la Física, Ed. Limusa, México, (1979).

[20] Gettys E.W., Física clásica y moderna, Ed. Impresa, España, (1998).

[21] Tipler, Física, Ed. Reverté S.A., Barcelona, $3^{\text {ra }}$ Edición (1995).

[22] Waftorsky, Max W., Introducción a la Filosofía de las ciencias, Ed. Alianza Universitaria, España, (1976).

[23] Veslin O. y Veslin J., Corriger des copies, Ed.Hachette, París, (1992).

[24] Moreira M.A., Atividade docente na universidade, Luzzatto editores ltda, Porto Alegre, (1985).

\section{Anexo}

\section{A. Problemáticas cualitativas integrado- ras del tipo biológica y/o ambiental}

$\mathrm{a}_{1}$ - En los desiertos de arena las temperaturas durante el día son muy elevadas y durante la noche muy bajas. A qué se debe esto?

$\mathrm{a}_{2^{-}}$Un fenómeno natural que permite la vida (animal y vegetal) en los lagos y estanques profundos en invierno, cuando están congelados, es que el hielo se forma de arriba hacia abajo y no de abajo hacia arriba, lo que da lugar a que en las profundidades el agua esté líquida y allí sobreviven los organismos. Como se explica está situación?

$a_{3}$ - Por qué hay menos peligro de daño para las cosechas en un día de frío repentino, si hay una buena cubierta de nieve sobre las cosechas?

$\mathrm{a}_{4}$ - Se han observado nubes oscuras de insectos que se forman sobre las copas de los árboles, en la puesta del sol. A qué se debe este fenómeno?

$a_{5}$ - Por qué se recomienda a las personas que los días soleados de verano vistan ropas claras? Lo notable de este hecho es que también se observa en los astronautas en el espacio, cuyos trajes son blancos o plateados; por qué?

$\mathrm{a}_{6^{-}}$El aumento de la concentración de ciertos gases contaminantes (Por ej.: Bióxido de Carbono) produce un incremento del efecto "Invernadero". En que consiste dicho fenómeno? Por qué conduce al calentamiento global del planeta?

$a_{7}$ - Por qué se registran diferencias de temperatura durante el día entre el agua de un lago y la superficie del terreno que la circunda? A su vez al ponerse el sol quién se enfría más rápidamente? Fundamente su respuesta.

$a_{8}$ - Por qué una persona desnuda en un ambiente a cero grados tirita?

$\mathrm{a}_{9}$ - Por qué una persona se sonroja al desarrollar una actividad física intensa?

$\mathrm{a}_{10}$ - En qué se basa el efecto refrescante que siente una persona durante el verano al colocarse en la corriente de aire generada por un ventilador? Qué sucede si el aire posee un mayor contenido de humedad?

\section{B. Problemáticas cualitativas del tipo académicas y/o naturales}

$\mathrm{b}_{1}$ - Por qué dos trozos de igual masa, uno de plomo y otro de hierro, inicialmente a igual temperatura y expuestos al sol por igual intervalo de tiempo, alcanzan temperaturas diferentes?

$\mathrm{b}_{2}$ - Por qué el agua no puede utilizarce como líquido termométrico?

$\mathrm{b}_{3}$ - Se dice que la escarcha formada sobre las tuberías que transportan amoníaco líquido a muy baja temperatura, obvian el uso de aislante para evitar su calentamiento. Por qué?

$\mathrm{b}_{4}$ - Considera una taza de té demasiado caliente para beberse. Si quitas la superficie superior del té es muy probable que puedas beber el resto. Como explicas este hecho?

$b_{5}$ - Por qué el interior de los termos está espejado?

$\mathrm{b}_{6}$ - Por que se calientan excesivamente los asientos de los autos colocados a los rayos del sol y con los vidrios transparentes cerrados?

$\mathrm{b}_{7}$ - Si para calentar la cama en una fría noche de invierno, tuviéramos que elegir entre $1 \mathrm{Kg}$ de agua en una bolsa de goma, o un cilindro de hierro de $1 \mathrm{Kg}$. Cuál de los dos resultaría mas efectivo? Argumenta tu elección.

$\mathrm{b}_{8}$ - Por que una barra de metal se calienta al golpearla con un martillo?.

$\mathrm{b}_{9}$ - Explica cuál es la función del radiador con agua de un automóvil.

$\mathrm{b}_{10^{-}}$Si tenemos 2 cubos de hielo de igual masa, uno sometido a una corriente de aire y el otro nó; cuál se derretirá primero? Qué sucede si el aire utilizado posee un mayor contenido de humedad?

Referencia: Los problemas de igual subindice ( $a_{i} y$ $\left.b_{i}\right)$ representan problemáticas de un mismo tópico, pero en un caso $\left(a_{i}\right)$ del tipo biológico-ambiental y en el otro $\left(b_{i}\right)$ del tipo académico. 